\title{
STUDY OF NASAL CARRIAGE OF MRSA AMONG POST GRADUATE STUDENTS IN A TERTIARY CARE HOSPITAL
}

Geeta S. $\mathrm{H}^{1}$, Rama N. K², Abhinandan $\mathrm{K}^{3}$

\section{HOW TO CITE THIS ARTICLE:}

Geeta S. H, Rama N. K, Abhinandan K. "Study of Nasal Carriage of MRSA among Post Graduate Students in a Tertiary Care Hospital". Journal of Evolution of Medical and Dental Sciences 2015; Vol. 4, Issue 55, July 09; Page: 9553-9560, DOI: 10.14260/jemds/2015/1381

ABSTRACT: BACKGROUND: Staphylococcus aureus is a common cause of community acquired and hospital acquired infections. One of the important sources of Staphylococci for nosocomial infections is the nasal carriage among hospital personnel. Emergence of drug resistant strains especially MRSA is a serious problem in hospital environment. Within the hospital, colonised healthcare workers act as reservoir for the spread of MRSA to uncolonised susceptible patients. Patterns of nasal carriage across the spectrum of health care settings are poorly characterised. The knowledge of prevalence rates associated with MRSA nasal carriage patterns could help in infection prevention strategies. The present study was undertaken to know the nasal carriage rates of MRSA among the newly joined batch of postgraduate students in our hospital by phenotypic and genotypic methods. MATERIALS \& METHODS: 29 PG students from various clinical departments of our hospital were screened for nasal carriage of MRSA as soon as they were admitted to the college and the same students were screened 6 months after exposure to the hospital atmosphere. MRSA isolates were identified by different phenotypic tests and confirmed by genotyping. RESULTS: Among the 29PG students, MRSA was isolated from one student in the swabs collected immediately after their admission. Hence the case was excluded from the study. Only 28 students were further studied. Among them none of them had MRSA nasal colonisation. 09 (32.1\%) MRSA were isolated in the same group of PGs after 6 months of repeat testing. Genotyping of all the MRSA (09) isolates showed the presence of mecA gene (100\%). CONCLUSION: Screening of the health care worker will give prevalence of MRSA carriers among health care workers who can be treated and further educated regarding spread of infection. Adherence to universal precautions can prevent spread of infections in the hospital.

KEYWORDS: Screening for MRSA carrier and Health care workers.

INTRODUCTION: Methicillin resistant Staphylococcus aureus (MRSA) is the commonly identified antibiotic resistant pathogen causing hospital acquired infection throughout the world in developing as well as developed countries; epidemiologic data is still incomplete \& not widely available.[1,2]

With a few exceptions, the incidence of nosocomial infections caused by MRSA continues to increase worldwide.[3] Infections caused by MRSA strains are associated with longer hospital stays, prolonged antibiotic administration \&association of other risk factors. Nasal colonisation with $\mathrm{S}$. aureus is a dynamic process; infected patients, contaminated environmental surfaces, colonised health care workers act as reservoirs for the spread of pathogen to patients.[1]

The nose is the main ecological niche where $S$. aureus colonises in human beings. Although other body sites like hands, skin, axilla \& intestinal tract can harbour MRSA; the anterior nares are the main reservoirs.[4,5] Eradication at this site prevents spread of infection to the patients admitted to high risk areas like ICUs, NICUs, PICUs and OTs etc. The association between S. aureus nasal carriage \& staphylococcal disease was first reported by Danbolt in 1931 who studied furunculosis.[6,7] 
The most important mode of transmission is through contaminated hands. An alternative mechanism of spread is airborne dispersal of staphylococci in association with an upper respiratory tract infection. ${ }^{[8,9]}$

Health care workers including post graduate students are most often transiently colonised, but they may become persistent carriers if they have dermatitis or sinusitis etc, and this may lead to prolonged MRSA carrier state \& hence transmission to the patients. The prevalence of MRSA colonisation among health care workers which was assessed extensively between $1980-2011$ in two reviews was found to be upto $50 \% \cdot{ }^{[8]}$

MRSA carriage rate in health care workers in Staphylococcal non-outbreak settings is thought to be higher than in outbreak situations, due to increased hygiene awareness in outbreaks.[7] The burden of health care associated MRSA colonisation seems to extend beyond the clinical settings to long term care facilities, outpatient care \& even to the community.[6]

Since the introduction of antibiotics in 1950's, there was considerable increase in the incidence of MRSA. These MRSA were initially usually resistant to numerous antibiotics, sometimes sensitive to rifampicin \& fusidic acid and were always sensitive to vancomycin.[10,11] Vancomycin resistance in MRSA is gradually emerging.[12,13]

MRSA can colonise the health care workers when exposed to the hospital atmosphere.[6] MRSA carriage rates will be higher when health care workers comply poorly with hand hygiene \& contact precautions. Identification of asymptomatic MRSA carriers by screening techniques is one of the most important measures to reduce the risk of nosocomial transmission and MRSA infections ${ }^{[14,15]}$

Decontamination of nasal colonised health care workers with topical mupirocin is recommended by most guidelines, but critical systematic use of this as an antibacterial agent still remains a question since emerging mupirocin resistant strains of MRSA are also reported.[8]

\section{AIMS \& OBJECTIVES:}

1. To study MRSA colonisation in clinical post graduate students, before exposure to the hospital environment\& after 6 months of working in the hospital atmosphere.

2. To detect the mecA gene in these MRSA strains isolated.

REJECTION CRITERIA: Clinical Post Graduate students who had signs and symptoms of infection; those who were on antibiotics recently and also with MRSA carrier state identified with first screening were excluded from the study group.

MATERIALS \& METHODS: The present study was carried out to know the prevalence of nasal carriage of S. aureus among newly joined clinical PG students in various clinical courses of our hospital from June 2014 - Jan 2015. They were screened at the time of joining the course (In June 2014) and screening was repeated after 6 months of working in their respective departments (Jan. 2015), to know the carrier status.

A total of 29 clinical PG students were screened for MRSA after obtaining informed consent and approval by the institute's ethical committee. Nasal swabs were collected from the anterior nares of both nostrils with a pre-moistened sterile swab in saline. Nasal swabs were processed within 2 hours of specimen collection; primary plating was done on blood agar (BA) \& Mannitol salt agar (MSA) and incubated at $37^{\circ} \mathrm{C}$ in ambient air $24-48$ hours. The isolates were identified by standard protocol. 


\section{ORIGINAL ARTICLE}

MSA contains Mueller-Hinton agar (MHA), $1 \%$ mannitol, $6.5 \% \mathrm{NaCl}$ and phenol red as indicator. The Phenol red detects mannitol fermenting colonies of $\mathrm{S}$. aureus which appear yellow colored.[16]

Antibacterial susceptibility testing (ABST) was performed on all the S. aureus isolates using Kirby Bauer disc diffusion method. The S. aureus ATCC 25923 was used as quality control strain. MRSA was detected using cefoxitin $(30 \mu \mathrm{g})$ in accordance with standards recommended by Clinical and Laboratory Standards Institute (CLSI) by commercially available antibiotic discs from Hi Media Pvt Ltd, India.[16,17]

DNA extraction was done using Amnion, Ampure Bacterial DNA Mini Spin Isolation Kit.

S. aureus isolates were cultured on blood agar overnight. Three to four bacterial colonies were suspended in $150 \mu \mathrm{l}$ sterile distilled water with lysostaphin $(1 \mathrm{mg} / \mathrm{ml})$ and incubated at $37^{\circ} \mathrm{C}$ for 30 mins according to the kit manufacturer's instructions. Extracted DNA was stored at $-20^{\circ} \mathrm{C}$ and used for PCR amplification.

Genotyping of the isolated MRSA strains was done by PCR for mecA genes.

The Oligonucleotide primers used for mecA PCR amplification for the identification of methicillin-resistance in S. aureus.[18]

\begin{tabular}{|c|c|c|c|}
\hline Primer & $\begin{array}{c}\text { Primer Sequence } \\
{\left[\mathbf{5}^{\prime} \mathbf{-} \mathbf{3}^{\prime} \mathbf{]}\right.}\end{array}$ & $\begin{array}{c}\text { Amplicon } \\
\text { size(bp) }\end{array}$ & $\begin{array}{c}\text { Nucleotide } \\
\text { Position }\end{array}$ \\
\hline MR 1 & GTAGAAATGACTGAACGTCCGAT & 310 & $318-342$ \\
\hline MR 2 & CCAATTCCACATTGTTTCGGTCT & 310 & $603-627$ \\
\hline
\end{tabular}

RESULTS: A total of 28nasal swabs from the newly joined post graduate students of various clinical departments were collected.

In 28 newly joined PG samples, S. aureus was isolated in 02 (7.14\%). In the same group (28) of PGs after 6 months 13 (46.2\%) S. aureus and 09 (69.2\%) MRSA were isolated. All the MRSA strains isolated in the PG students after 6 months of exposure to the hospital atmosphere showed the presence of mecA gene 09 (100\%).

Table I shows the distribution of various organisms isolated in the nasal screening of PG's. The organisms isolated other than Staphylococci were Klebsiella sps (07) \& (03) in pre exposed and the exposed PGs; Citrobacter sps (01) in pre exposed; Proteus sps (01) in the exposed PGs and Eshericia coli (01) in pre exposed. The bacteria isolated other than S. aureus (Klebsiella sps, Citrobacter sps, Proteus sps \& Eschericia coli) were sensitive strains.

Table II shows the department-wise isolation of Staphylococci in the nasal screening of newly joined PG's.

Table III shows the prevalence of nasal carriage rate of S. aureus and MRSA.

Multiplex PCR assay done on the nasal screening MRSA isolates showed a $100 \%$ presence of mecA gene. 


\section{ORIGINAL ARTICLE}

\begin{tabular}{|c|c|c|}
\hline \multirow{2}{*}{ Organisms } & Pre Exposed (28) & Exposed (28) \\
\cline { 2 - 3 } & [June 2014] & [Jan 2015] \\
\hline MRSA & -- & 09 \\
\hline MSSA & 02 & 04 \\
\hline CONS & -- & 01 \\
\hline ASBs & 06 & 05 \\
\hline Klebsiella sps & 07 & 03 \\
\hline Citrobacter sps & 01 & -- \\
\hline Proteus sps & -- & 01 \\
\hline Escherichia coli & 01 & -- \\
\hline No growth & 11 & 05 \\
\hline
\end{tabular}

Table 1: Organisms isolated in MRSA screening of PG students

\begin{tabular}{|c|c|c|c|c|c|c|}
\hline Department & \multicolumn{2}{|c|}{ MRSA (09) } & \multicolumn{2}{c|}{ MSSA (06) } & \multicolumn{2}{c|}{ CONS (01) } \\
\hline & Pre-Exposed & Exposed & Pre Exposed & Exposed & Pre Exposed & Exposed \\
\hline Medicine & -- & 03 & 01 & 03 & -- & -- \\
\hline Paediatrics & -- & 03 & 01 & 01 & -- & -- \\
\hline Surgery & -- & 01 & -- & -- & -- & -- \\
\hline Ophthalmology & -- & 02 & -- & -- & -- & -- \\
\hline OBG & -- & -- & -- & -- & -- & 01 \\
\hline
\end{tabular}

Table 2: Prevalence of S. aureus with respect to clinical departments

\begin{tabular}{|c|c|c|}
\hline Staphylococcal sps & $\begin{array}{c}\text { Nasal carriage rate } \\
\text { (At Admission to PG course) }\end{array}$ & $\begin{array}{c}\text { Nasal carriage rate (After 6 } \\
\text { months of working in hospital ) }\end{array}$ \\
\hline S. aureus & $02 / 28(7.14 \%)$ & $13 / 28(46.4 \%)$ \\
\hline MRSA & -- & $09 / 13(69.2 \%)$ \\
\hline
\end{tabular}

Table 3: Nasal carriage rate of S. aureus and MRSA

Multiplex PCR assay done on the nasal screening MRSA isolates showed a $100 \%$ presence of mecA gene.

\begin{tabular}{|c|c|c|}
\hline MRSA Genotype & $\begin{array}{c}\text { MRSA (Nil) } \\
\text { (At Admission to PG course) }\end{array}$ & $\begin{array}{c}\text { MRSA (09) } \\
\text { (After 6 months of working in hospital) }\end{array}$ \\
\hline mecA & -- & $09(100 \%)$ \\
\hline \multicolumn{2}{|c|}{ Table 4: Genotyping statistics of isolated MRSA strains } \\
\hline
\end{tabular}




\section{ORIGINAL ARTICLE}

\section{Fig I: PCR Results}

Samples: 11

Primer : MF1 MF2

Sequence : mecA gene

GTAGAAATGACTGAACGTCCGAT CCAATTCCACATTGTTTCGGTCT

Product size : $310 \mathrm{bp}$

PC- Positive Control : Staph ATCC

NC- Negative Control

RESULTS :

$1 \mathrm{a}, 1 \mathrm{~b}, 2,3,4,5,6,7,8,9, \mathrm{NC}, \mathrm{PC}, 10$

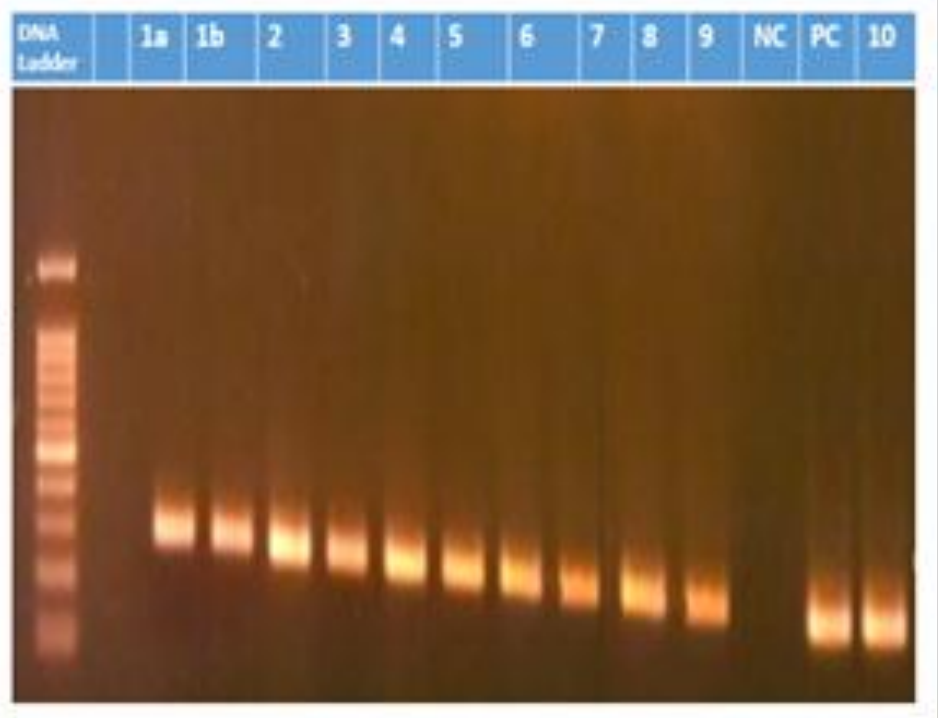

DISCUSSION: Staphylococcus aureus is a frequent cause of nosocomial infections amounting to twenty five percent. S. aureus nasal carriage among healthy adults ranges from approximately 20$30 \%$, with higher prevalence seen in overcrowded populations.[14,19,20]

In the present study, there was considerable increase in the nasal carriage rate of $S$. aureus (46.4\%) \& MRSA (69.2\%) in PG screening done 6 months after exposure to the hospital environment. The overall nasal carriage rate of S. aureus in the pre exposed (7.14\%). [Table III]

Studies by Agumas Shibabaw et al., 2013[1] \& Mohammad Kazem Shariffi Yazdi et al., 2009[19] showed lower nasal carriage rates of S. aureus (28.8\% and $12.7 \%)$ \& MRSA $(12.7 \%$ and $11.4 \%)$ respectively. Whereas, Shukla I et al., 2011[3] showed a higher prevalence of S. aureus (78.6\%) \& Bushra Ashiq et al., 1989[10] reported the lowest MRSA carriage rate of $4 \%$.

The S. aureus and MRSA carriage rates depend on various factors related to the hospital environment, associated infections in the hospital personnel $\&$ the practices concerning universal precautions.

Among the PGs exposed to hospital environment for 6 months, the carriage rate was almost equal in both surgical - allied (04/28) and medical- allied (05/28) departments, amounting to a total of 09/13 MRSA strains.[Table II]

A few studies have shown a preponderance of MRSA isolation in the surgical departments.[2,10,21] Reports from western countries such as France ${ }^{[21]}$ and Spain,[22] shows very high rate $(45.4 \%, 34.9 \%$ and $38 \%, 84 \%)$ for S. aureus and MRSA prevalence respectively in hospital personnel. Du J et al. 2010 reported the nasal carriage rate of $15.4 \%$ for S. aureus and 19.4\% for MRSA. All MRSA isolates tested were susceptible to vancomycin, quinupristin/dalfopristin, teicoplanin and linezolid.[23]

In the present study, $80 \%$ of MRSA were found to be resistant to erythromycin; $60 \%$ to ampicillin; $50 \%$ to clindamycin and $40 \%$ to cotrimoxazole. All the isolated strains of MRSA were sensitive to linezolid, teicoplanin and vancomycin.

The molecular characterisation by other authors reported data on mecA, PVL genes, SCC mec typing and spa typing in MRSA isolates was comparable.[23,24] In the present study, only mecA 
genotyping was done. All the isolated MRSA strains showed the presence of mecA gene.[Table IV] [Fig. 1]

CONCLUSION: The major reservoir of methicillin resistant staphylococci (MRSA) in hospitals is colonised health care workers \& colonised or infected in-patients. All groups of health care workers should be screened regularly and at frequent intervals to know the real burden of MRSA colonisation. Strict infection control measures can effectively control MRSA transmission in health care settings.

\section{REFERENCES:}

1. Shahibabaw Agumas, Tamrat Abebe and Adane Mihret. Nasal carriage rate of methicillin resistant Staphylococcus aureus among Dessie referral hospital health care workers; Dessie, Northeast Ethiopia. Antimicrob Resist Infect Cont. 2013; 2:25.

2. Ahmed MO, Elramalli AK, Amri SG, Abuzweda AR and Abouzeed YM: Isolation and screening of methicillin resistant Staphylococcus aureus from health care workers in Libyan hospitals. East Meditrr Health J. 2012; 18: 37 - 42.

3. Kumar P, Shukla I, and Varshney S: Nasal screening of health care workers for coagulase positive MRSA and nasal colonisation with Staphylococcus aureus. Biology and Medicine. 2011; 3: $182-6$.

4. Madeleian Dulon, Claudia Peters, Anja Schablon and Albert Neinhaus. MRSA carriage among health care workers in non - outbreak settings in Europe and The United States: a systematic reviews. BMC Infect Dis. 2014; 14:363.

5. European Antimicrobial resistance Surveillance network: Antimicrobial resistance surveillance in Europe. Annual report of the European Antimicrobial Resistance Surveillance Network (EARS-Net) 2012. Stockholm. ECDC; 2013.

6. Heiman F L Wertheim, Melles DC, Van Leeuwen W, Van Belkum A, Henri A Verburgh and Jan L Nouwen.The role of nasal carriage in Staphylococcus aureus infections. Lancet Infect Dis. 2005; 5: $751-762$.

7. Solberg CO. A study of carriers of Staphylococcus aureus with special regard to quantitative bacterial estimations. Acta Med Scand Suppl. 1965; 436: 1-96.

8. Dulon M, Haamaan F, Peters C, Schablon A and Neinhaus A: MRSA prevalence in European health care settings: a review. BMC Infect Dis. 2011; 11: 138.

9. Albrich WC and Harbarth S: Health care workers: source, vector or victim of MRSA? Lancet Infect Dis. 2008; 8: 289 - 301.

10. Bushra Ashiq, Abdul Sattar, Zermina R and Khan JA. The carrier state: methicillin - resistant Staphylococcus aureus a hospital study "screening of hospital personnel" for nasal carriage of S. aureus. JPMA. 1989; 39: 35 - 8.

11. White, A. Relation between quantitative nasal cultures and dissemination of staphylococci. Lab. Clin. Med. 1961; 58: 273.

12. Venubabu Thati, Shivannavar CT and Gaddad SM. Vancomycin resistance among MRSA isolates from ICUs of tertiary care hospitals in Hyderabad. IJMR. Nov 2011; 134 (5): 74 - 8. DOI: 10.4103/0971-5916.91001.

13. George Sakoulas and Robert C. Moellering, Jr. increasing antibiotic resistance among MRSA strains. Clin Infect Dis. 2008; 46 (Supplement 5): S360 - S367. DOI: 10.1086/533592. 


\section{ORIGINAL ARTICLE}

14. Philipp Warnke, Tim Harnack, Peter Ottl, Guenther Kundt and Andreas Podbielski. Nasal Screening for Staphylococcus aureus - Daily Routine with Improvement Potentials. PLOS ONE. February 2014; 9: 2. e89667.

15. Weidenmaier C, Goerke C and Wolz C. Staphylococcus aureus determinants for nasal colonization. Trends Microbiol. 2012; 20: 243-50.

16. Mackie \& MacCartney. Practical Medical Microbiology. New York, Churchill Livingstone, 1996; 14th Ed: $249-50$.

17. Clinical and Laboratory Standard Institute. Performance standards for antimicrobial susceptibility testing; Twenty-third informational Supplement. CLSI document M100-S23. Wayne, PA: CLSI; 2013.

18. Protocol for PCR amplification of mecA, mecC (mecA LGA251), PVL and spa recommended by the EURL-AR, DTU Food, National Food Institue; 2st version, september 2012.

19. Mohammad Kazem Sharifi-Yazdi, Mohammad Bagher Khalili, Hosein Dargahi and Hosein Ali Sadeghian. Nasal Colonization rate of Staphylococcus aureus strains among Health Care Service Employees of Teaching University Hospitals in Yazd. Acta Medica Iranica. 2009; 47(4): 315 - 7.

20. Kluytmans J, Van belkum A and Vergrugh H. Nasal carrigae of Staphylococcus aureus: epidemiology, underlying mechanisms and associated risks. Clin Microbiol Rev. 1997; 10: 505-20.

21. Akoua Koffi C, Dje K, Toure R, Guessennd N, Acho B and Faye Kette H. Nasal carriage of meticillin-resistant Staphylococcus aureus among health care personnel in Abidjan (Côte d'lvoire). Dakar Med. 2004; 49: 70 - 4.

22. Tejero A, Gutiérrez MA, Aiquel MJ, Brandago M, González C and Broussain MT. Nasal carriage of Staphylococcus aureus among personnel working in a teaching hospital. Enferm Infect Microbiol Clin. 1991; 9: 351 - 3.

23. Du Jimei, Chen Chun, Ding Baixing, Tu Jinjing, Qin Zhiqiang, Parsons Chris et al. (2011) Molecular Characterization and Antimicrobial Susceptibility of Nasal Staphylococcus aureusIsolates from a Chinese Medical College Campus. PLoS ONE 6(11): e27328. doi:10.1371/journal.pone.0027328.

24. Kang YC, Tai WC, Yu CC, Kang JH and Huang YC. MRSA nasal carriage among patients receiving haemodialysis in Taiwan: prevalence rate, molecular characterisation \& decolonisation. BMC Infect Dis. 2012; 12: 284. 


\section{ORIGINAL ARTICLE}

\section{AUTHORS:}

1. Geeta S. H.

2. Rama N. K.

3. Abhinandan K.

\section{PARTICULARS OF CONTRIBUTORS:}

1. Associate Professor, Department of Microbiology, MVJ Medical College \& Research Hospital, Hoskote, Bangalore, India.

2. HOD, Department of Microbiology, MVJ Medical College \& Research Hospital, Hoskote, Bangalore, India.

FINANCIAL OR OTHER

COMPETING INTERESTS: None
3. Professor, Department of Microbiology, MVJ Medical College \& Research Hospital, Hoskote, Bangalore, India.

\section{NAME ADDRESS EMAIL ID OF THE} CORRESPONDING AUTHOR:

Dr. Geeta S. H,

No 54/10, Arun Nivas Apartments,

R. M. S. Colony, Bhattarahalli, Old Madras Road,

Bangalore-560049.

E-mail: drgeetashashikant@gmail.com

Date of Submission: 16/06/2015.

Date of Peer Review: 17/06/2015.

Date of Acceptance: 01/07/2015.

Date of Publishing: 07/07/2015. 